\title{
Correction factor between two calcitonin assays: DiaSorin LiaisonXL and Cobas E601
}

\author{
Mara Baetu ${ }^{1,2^{*}}$, Alexandru Draghici ${ }^{3}$, Adriana Padure 4 , Stefana Bucur ${ }^{2,5}$, Andra \\ Caragheorgheopol ${ }^{2,4}$, Corin Badiu ${ }^{1,2}$
}

\author{
1. Thyroid II, “C.I. Parhon” National Institute of Endocrinology, Romania \\ 2. "Carol Davila” University of Medicine and Pharmacy, Romania \\ 3. Mathematics and Informatics Faculty, University of Bucharest, Romania \\ 4. Research Laboratory, “C.I. Parhon” National Institute of Endocrinology, Romania \\ 5. Colentina Clinical Hospital, Romania
}

\begin{abstract}
Introduction: Calcitonin measurement is important for the diagnosis and monitoring of medullary thyroid carcinoma. Unfortunately, in clinical practice, different detection systems assays are used in the follow-up of the patients, which can be misleading. Objective: To identify the correction factor for calcitonin measurement on two different immunoanalysers: DiaSorin-LiaisonXL (immunochemiluminescence) and CobasE601 (electro-immunochemiluminescence). Methods: We selected 89 registered CT samples (28-from men; 61-from women), that were analysed on CobasE601 with reported values between $0.5 \mathrm{pg} / \mathrm{ml}$ and $2812 \mathrm{pg} / \mathrm{ml}$ (128.5 513.98$)$. These CT samples were selected randomly to cover as wide a range of values as possible, and represented either basal CT $(n=38)$ or selected from CT stimulation tests $(n=51)$. Samples were evaluated subsequently on DiaSorin-LiaisonXL. All patients gave their informed consent. Results: Between the two assays a segmented linear correlation was noted. We identified the following general linear regression equation: $1.108 x+19.337(p<0.05)$. The bias increased at high calcitonin values. Therefore, for a better accuracy we analysed the regression equation segmentally. A statistic difference $(p<0.05)$ was noted for CT values ranged between 350-2600 pg/ml $(n=31)$ on DiaSorin-LiaisonXL, for which the linear regression equation for CobasE601 becomes 1.009x+169.796. Conclusions: Calcitonin correction factors are highly important in the dynamic follow up of a patient suffering from medullary thyroid carcinoma when different detection systems assays are used to determine calcitonin. We identified the correction factors for calcitonin determination between two different frequently used chemiluminescence immunoanalysers: DiaSorin-LiaisonXL and CobasE601. However, it is strongly advisable to use the same analyser in order to establish biochemical evolution of calcitonin.
\end{abstract}

Keywords: medullary thyroid carcinoma, calcitonin, Cobase601, LiaisonXL, correction factor Received: 13 ${ }^{\text {th }}$ June 2021; Accepted: 13 ${ }^{\text {th }}$ July 2021; Published: $16^{\text {th }}$ July 2021

\footnotetext{
* Corresponding author: Mara Baetu, Thyroid II, “C.I. Parhon” National Institute of Endocrinology, Romania. E-mail:mara.baetu@gmail.com
} 


\section{Introduction}

Calcitonin (CT) is a hormone produced by the parafollicular C-cells of the thyroid gland (1). $\mathrm{CT}$ levels tend to be raised under the age of 3 years (2) the timing of which is based on the mutation-associated risk and the calcitonin (CT, mainly in the first 6 months of life (3), after which they decline rapidly and are relatively stable from childhood through adult life (4). These values are higher in men compared with women, probably due to the presence of a larger C-cell mass (3).

The physiological role of CT is still in question in humans, but it has proven efficiency being used as treatment in osteoporosis or hypercalcemia (1). The main reason why CT has clinical interest is by virtue of its role as a tumour marker in the diagnosis and dynamic follow-up of medullary thyroid carcinoma (MTC) (5). CT stimulation tests with calcium could increase the sensitivity of CT analysis $(3,6)$.

Increased $\mathrm{CT}$ values can also be found in various other thyroid or non-thyroid related conditions such as: autoimmune thyroiditis, hyperparathyroidism, hypercalcemia, hypergastrinemia, acute pulmonary inflammatory conditions, chronic inflammatory disease, mastocytosis, neuroendocrine tumours, small and large cell pulmonary carcinoma, breast cancer, prostate cancer, leukemic and myeloproliferative disorders $(3,7-9)$ supplemented with additional published materials, and then created evidence-based recommendations, which were set in categories using criteria adapted from the United States Preventive Services Task Force Agency for Healthcare Research and Quality. The original guidelines provided abundant source material and an excellent organizational structure that served as the basis for the current revised document. Results: The revised guidelines are focused primarily on the diagnosis and treatment of patients with sporadic medullary thyroid carcinoma (MTC. Treatment with proton pump inhibitors, beta-blockers or glucocorticoids can also raise serum CT (3). CT is metabolized by the kidney and liver, therefore conditions such as chronic kidney disease can result in elevated CT values (3).

The progression disease and aggressiveness of an MTC can be predicted by measuring serum CT and carcinoembryonic antigen (CEA) at different moments over a period of time to determine their doubling time (10). The amount of time at which CT value doubles (CT doubling time) can also be a sensitive indicator of a right moment to initiate tyrosine-kinase inhibitors (11). Therefore, it becomes utterly important to monitor CT on the same detection system. Current guidelines recommend evaluating the patient with the same assay throughout the entire monitoring period, before and after the surgery, due to variability in CT determination in different commercial assays that have various normal reference values reported (3). Unfortunately, in clinical practice, throughout one patient follow-up, different detection system assays are used, which can be misleading and makes the monitoring difficult.

\section{Objective}

We aimed to establish the correction factor for CT measurement on two different frequently used automatic immunoanalysers: DiaSorin LiaisonXL (immunochemiluminescence) and Cobas E601 (electro-immunochemiluminescence).

\section{Patients and methods}

We selected 89 registered CT samples (28 from men, 61 from women) that were analysed on Cobas E601 with reported values between $0.5 \mathrm{pg} /$ $\mathrm{ml}$ and $2812 \mathrm{pg} / \mathrm{ml}(128.5 \pm 513.98)$. These 89 values were selected randomly to cover as wide a range of values as possible, and represented either basal CT $(n=38)$ or selected from various $\mathrm{CT}$ stimulation tests $(\mathrm{n}=51)$ (not all samples from a singular stimulation test could be selected due to economic reasons). The 89 serum samples 
were collected between 8-10 AM after overnight fasting. The stimulated CT samples belonged to patients with high basal CT values to whom we performed a calcium stimulation test to investigate hypercalcitoninemia. Briefly, after cardiological assessment, patients were intravenously injected within 3-5 $\mathrm{min} 2.47 \mathrm{mg} / \mathrm{KgBW}$ (kilogram of body weight) of elemental calcium, adjusted to the patient's ideal weight, using calcium gluconate. The stimulated CT samples were collected after $\mathrm{Ca}$ administration at 2, 5, and 10 minutes.

These samples were obtained from patients evaluated in Thyroid II Department and Research Laboratory of C.I. Parhon National Institute of Endocrinology, Bucharest, Romania, between June and July 2018. An informed consent was obtained according to current ethical standards. Study approval was provided by the Ethics Committee of Carol Davila University of Medicine and Pharmacy, Bucharest, No. 161/ PO-35-F-03/14.06.2018. All procedures were in agreement with the Helsinki declaration concerning ethical principles for medical research involving human subjects.

On the initial analysis on Cobas E601, the 2 samples that were above the assay measuring range (2000 pg/ml) were diluted $1 / 100$ and recalculated. Afterwards, all samples were aliquoted and stored at $-20^{\circ} \mathrm{C}$. At the time of the second analysis (not later than 3 months from the initial sample), we thawed these samples and subsequently analysed them on DiaSorin LiaisonXL assay. Also, the 2 samples that were above the DiaSorin LiaisonXL CT assay measuring range $(2000 \mathrm{pg} / \mathrm{ml})$ were measured after dilution $1 / 100$ and recalculated.

Furthermore, a statistical analysis was performed using IBM SPSS Statistics version 20.

\section{Calcitonin immunoassays}

In this study, for the quantitative determination of CT in human serum we used two immunoche- miluminescence assays on two automated analysers: DiaSorin LiaisonXL and Cobas E601.

The DiaSorin LiaisonXL CT assay represents a direct, two-site, one-step sandwich chemiluminescence immunoassay (CLIA). Affinity-purified mouse antibody to the synthetic human CT, coated to the solid phase, and the second affinity-purified mouse antibody conjugated to an isoluminol derivative are incubated. After the incubation, the unbound material is removed, the starter reagents are added and a flash chemiluminescent reaction is initiated. The light signal is measured by a photomultiplier and is proportional to the concentration of CT present in calibrators, controls, and samples. The measuring range of the assay is between $1 \mathrm{pg} / \mathrm{ml}$ and 2000 $\mathrm{pg} / \mathrm{ml}$; limit of detection is $\leq 0.1 \mathrm{pg} / \mathrm{ml}$, limit of quantitation is $\leq 3.0 \mathrm{pg} / \mathrm{ml}$. Any samples higher than $2000 \mathrm{pg} / \mathrm{ml}$ should be manually diluted in the specific diluent (recommended dilution ratio $1 / 100)$. The reference values are: $1-4.8 \mathrm{pg} /$ $\mathrm{ml}$ for women, and 1-11.8 pg/ml for men. In our hands, for normal and pathological control levels, CV\% was $7.79 \%$, and $7.30 \%$, respectively. It is also mentioned that the cross-reactivity for CGRP (CT gene-related peptide) and procalcitonin is $<0.01 \%$ (12).

Cobas E601 CT assay represents an electrochemiluminescence immunoassay (ECLIA) based on two-steps sandwich principle that engages monoclonal antibodies specifically directed against human CT, labelled with ruthenium complex and biotin, respectively. In the measuring cell, the microparticles of the aspirated mixture are magnetically captured onto the surface of the electrode, and the unbound substances are removed. Application of a voltage to the electrode induces the chemiluminescent emission which is measured by a photomultiplier and the results are determined on a calibration curve. Limit of detection is $0.5 \mathrm{pg} / \mathrm{ml}$ and limit of quantitation is $1.0 \mathrm{pg} / \mathrm{ml}$. The measuring range of the assay is between $0.5 \mathrm{pg} / \mathrm{ml}$ and $2000 \mathrm{pg} / \mathrm{ml}$. Any samples 
higher than $2000 \mathrm{pg} / \mathrm{ml}$ should be automated or manually diluted in the specific diluent (recommended dilution ratio $1 / 100$ ). The reported reference values are: $5.17-9.82 \mathrm{pg} / \mathrm{ml}$ for women, and $8.31-14.3 \mathrm{pg} / \mathrm{ml}$ for men. In our hands, for normal and pathological control levels, CV\% was $4.46 \%$, and $4.24 \%$, respectively. The reported cross-reactivity for CGRP is of $0.002 \%$ (13). Both assays are referenced to the World Health Organization CT International Standard (WHO 89/620) (12,13).

\section{Results}

The 89 registered CT samples that were initially analysed on Cobas E601 had reported values between $0.5 \mathrm{pg} / \mathrm{ml}$ and $2812 \mathrm{pg} / \mathrm{ml}$ (128.5 \pm 513.98). After CT samples were analysed on DiaSorin Liaison, the reported values were between $<1$ and $2600 \mathrm{pg} / \mathrm{ml}$. We found a strong Spearman correlation coefficient of 0.992 between DiaSorin LiaisonXL and Cobas E601. Between the two assays, a segmented linear correlation was noted. We identified the following general linear regression equation: $1.108 \mathrm{x}+19.337(\mathrm{p}<0.05)$.
When CT on DiaSorin LiaisonXL increases by 1 unit, CT on Cobas E601 increases by $1.108+$ 19.337. We observed the bias increased at high CT values. Therefore, for a better accuracy, we analysed the regression equation segmentally. A statistical difference $(\mathrm{p}<0.05)$ was noted for CT values ranged between $350-2600 \mathrm{pg} / \mathrm{ml}(\mathrm{n}=31)$ on DiaSorin LiaisonXL, for which the linear regression equation for Cobas E601 becomes $1.009 x+169.796$ (Figure 1).

\section{Discussion}

Over time, laboratory methods for determining CT have greatly evolved, from radioimmunoassays (RIA) to enzyme immunometric assays (EIA) and fluorescence immunoassays (FIA). The newest assays are based on CLIA (14). They are highly sensitive and specific for monomeric $\mathrm{CT}$ and they eliminate the cross-reactivity with other CT related peptides such as procalcitonin, which is of great importance in general inflammatory conditions such as sepsis $(3,15,16)$ supplemented with additional published materials, and then created evidence-based recommenda-

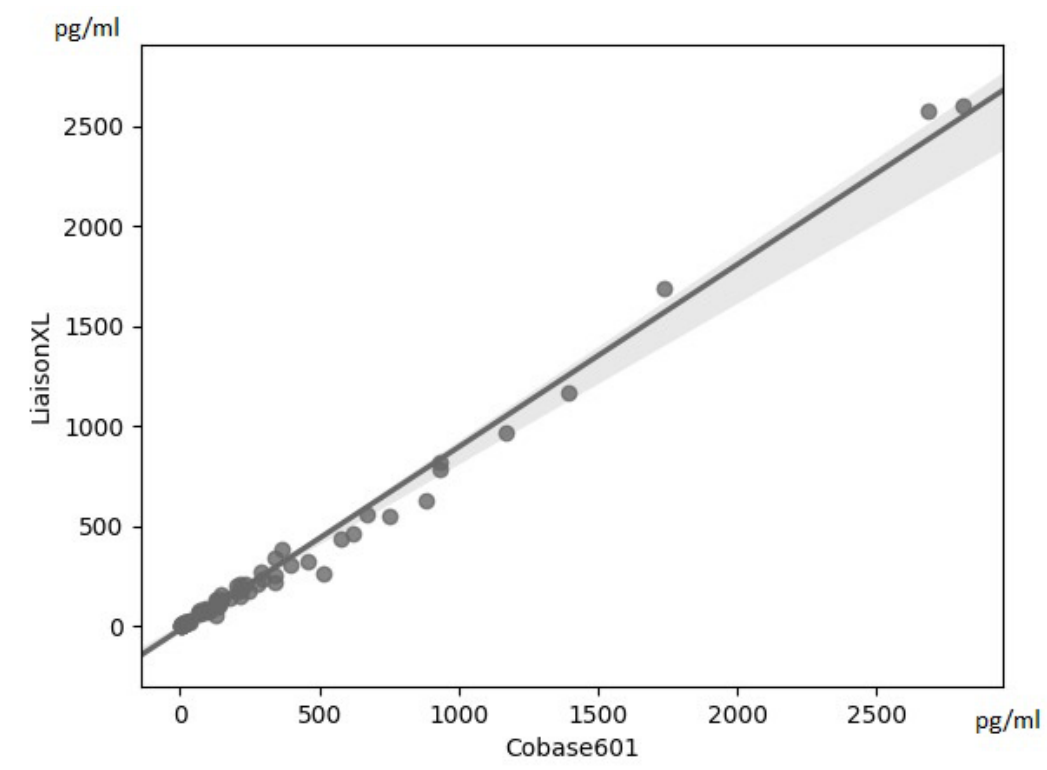

Fig. 1 Regression line (CobasE601-LiaisonXL) 
tions, which were set in categories using criteria adapted from the United States Preventive Services Task Force Agency for Healthcare Research and Quality. The original guidelines provided abundant source material and an excellent organizational structure that served as the basis for the current revised document. Results: The revised guidelines are focused primarily on the diagnosis and treatment of patients with sporadic medullary thyroid carcinoma (MTC. Also, CLIAs decrease the chance of the "hook effect" (3). The "hook effect" may occur when the analysed serum contains very high levels of CT that saturate the binding capacity of the antibody, resulting in misleading, falsely lower reported values $(3,17)$. There are controversies regarding the dosing of $\mathrm{CT}$ in the screening of thyroid nodules. Even though the American Thyroid Association (ATA) has not taken a position for or against CT screening, some reports have outlined the importance of CT measurement for thyroid nodules for early diagnosis of MTC $(3,18)$. Whether basal or stimulated, the measurement of CT is essential not only for the diagnosis of MTC, but also for its dynamic follow-up. Over the past years, the demand for CT dosage has been on an ascending trend in National Institute of Endocrinology C.I Parhon. We agree with ATA that the usage of different commercial assays for CT determination can be misleading in monitoring a patient. That is why we found it utterly necessary to establish a correction factor between two different frequently used CLIAs: DiaSorin LiaisonXL and Cobas E601.

We propose the following conversion formula from DiaSorin LiaisonXL to Cobas E601:

- for conversion of DiaSorin LiaisonXL CT values $\geq 350 \mathrm{pg} / \mathrm{ml}$ :

$$
\text { Cobas E601 value }=\text { LiaisonXL value }+170
$$

Reversely, from Cobas E601 to DiaSorin LiaisonXL, the proposed formula becomes:
- for conversion of Cobas E601 CT values $\geq 520 \mathrm{pg} / \mathrm{ml}$ :

DiaSorin LiaisonXLvalue $=$ Cobas value -170

A limitation to our study is that the results could be altered by the fact that the DiaSorin LiaisonXL analysis was performed on previously stored samples. Repeated freeze-thaw cycles may affect the test results (12). CT samples are stable for $19-24$ hours at $2-8^{\circ} \mathrm{C}$, while at room temperature the stability time decreases within only 2-4 hours. This is the reason why the samples should be stored frozen $\left(-20^{\circ} \mathrm{C}\right.$ or below), in glass or plastic vials. After thawing, the samples should be mixed well before (re)testing, but manufacturers recommend avoiding repeated freeze-thaw cycles $(12,13)$. Another limitation is represented by the small number of samples, determined by economic restrictions, which can bring bias to the correction factor. Carrying on the analysis on larger batches of freshly drawn samples will ensure an increased accuracy of these correction factors. Also, the measurement uncertainty determined in the laboratory for both methods was not taken into account. However, even if the value of the correction factor would undergo discrete changes, this would not influence the main aspect that we want to emphasize, namely the importance of taking the correction factors into account when evaluating a patient with different methods.

\section{Conclusions}

CT correlation factors are highly important in the dynamic follow-up of a patient suffering from MTC when different detection system assays are used to determine CT. To the best of our knowledge, this is the first study in literature that identifies the correction factors for CT determination between two different frequently used CLIAs: DiaSorin LiaisonXL CT assay and 
Cobas E601 CT assay. However, it is strongly advisable to use the same analyser in order to establish biochemical evolution of this tumour marker of MTC.

\section{Authors' contributions}

$\mathrm{MB}, \mathrm{SB}, \mathrm{AC}$ and $\mathrm{CB}$ researched literature and conceived the study. MB, AP, AC, and CB were involved in protocol development, operational completion of the study, data interpretation, gaining ethical approval and patient recruitment. ȘB and AD were involved in data analysis. MB wrote the first draft of the manuscript. All authors critically reviewed and approved the final version of the manuscript.

\section{Conflict of interests}

The authors declare that there is no conflict of interest.

\section{References}

1. Gardner DG, Shoback MD. Metabolic bone disease. In: Greenspan's Basic \& Clinical Endocrinology, Tenth Edition. Ohio: McGraw-Hill; 2018. p. 239-297.

2. Castagna MG, Fugazzola L, Maino F, Covelli D, Memmo S, Sestini F, et al. Reference range of serum calcitonin in pediatric population. J Clin Endocrinol Metab. 2015 May;100(5):1780-4. DOI: 10.1210/jc.2014-4508

3. Wells SA, Asa SL, Dralle H, Elisei R, Evans DB, Gagel $\mathrm{RF}$, et al. Revised American thyroid association guidelines for the management of medullary thyroid carcinoma. Thyroid. 2015 Jun;25(6):567-610. DOI: 10.1089/ thy.2014.0335

4. Eckelt F, Vogel M, Geserick M, Kirsten T, Bae YJ, Baber R, et al. Calcitonin measurement in pediatrics: Reference ranges are gender-dependent, validation in medullary thyroid cancer and effects of thyroid diseases. Clin Chem Lab Med. 2019 Jun;57(8):1242-50. DOI: 10.1515/cclm-2018-1186

5. Danila R, Livadariu R, Branisteanu D. Calcitonin revisited in 2020. Acta Endocrinol (Buchar) 2019 OctDec;15(4):544-8. DOI: 10.4183/aeb.2019.544

6. Băetu M, Dobrescu R. Novel markers for early diagnosis and prognostic classification in medullary thyroid carcinoma. Acta Endocrinol (Buchar). 2017 Oct;13(4):519-522. DOI: 10.4183/aeb.2017.519

7. Hillyard CJ, Oscier DG, Foa R, Catovsky D, Gold- man JM. Immunoreactive calcitonin in leukaemia. Br Med J. 1979 Dec;2(6202):1392-3. DOI: 10.1136/ bmj.2.6202.1392

8. Schwartz KE, Wolfsen AR, Forster B, Odell WD. Calcitonin in nonthyroidal cancer. J Clin Endocrinol Metab. 1979 Sep;49(3):438-44. DOI: 10.1210/jcem-49-3438

9. Toledo SPA, Lourenço DM, Santos MA, Tavares MR, Toledo RA, Correia-DeurI JE de M. Hypercalcitoninemia is not pathognomonic of medullary thyroid carcinoma. Clinics. 2009 Jul;64(7):699-706. DOI: 10.1590/ S1807-59322009000700015

10. Barbet J, Campion L, Kraeber-Bodéré F, et al. Prognostic impact of serum calcitonin and carcinoembryonic antigen doubling-times in patients with medullary thyroid carcinoma. J Clin Endocrinol Metab 2005 Nov;90(11):6077-6084. DOI: 10.1210/jc.2005-0044

11. Ito Y, Miyauchi A, Kihara M, et al. Calcitonin doubling time in medullary thyroid carcinoma after the detection of distant metastases keenly predicts patients' carcinoma death. Endocr J 2016 Jul;63(7):663-7. DOI: 10.1507/endocrj.EJ16-0140

12. LIAISON $®$ XL | DiaSorin. Available from: https:// www.diasorin.com/en/immunodiagnostic-solutions/ systems/clia-systems/liaisonr-xl. Accessed Mar 19, 2021.

13. Elecsys ${ }^{\circledR}$ Calcitonin. Available from: https://diagnostics.roche.com/global/en/products/params/elecsys-calcitonin.html. Accessed Mar 19, 2021.

14. Kahaly GJ, Algeciras-Schimnich A, Davis TE, Diana T, Feldkamp J, Karger S, et al. United States and european multicenter prospective study for the analytical performance and clinical validation of a novel sensitive fully automated immunoassay for calcitonin. Clin Chem. 2017 Sep;63(9):1489-96. DOI: 10.1373/ clinchem.2016.270009

15. Kratzsch J, Petzold A, Raue F, Reinhardt W, Bröcker-Preuß M, Görges R, et al. Basal and stimulated calcitonin and procalcitonin by various assays in patients with and without medullary thyroid cancer. Clin Chem. 2011 Mar;57(3):467-74. DOI: 10.1373/ clinchem.2010.151688

16. Shi J, Shi Q, Yuan S, Zhang W. Relationships between serum procalcitonin level, severity and different stresses of non-septic critically ill patients. Rev Romana Med Lab. 2020;28(1):49-56. DOI:10.2478/rrlm-2020-0003

17. Vashist SK, Luong JHT. Handbook of Immunoassay Technologies: Approaches, Performances, and Applications. London: Academic Press; 2018. DOI: 10.1016/ B978-0-12-811762-0.05001-5

18. Ross DS. Medullary thyroid cancer: Surgical treatment and prognosis - UpToDate Available from: https://www.uptodate.com/contents/diagnostic-approach-to-and-treatment-of-thyroid-nodules. Accessed Mar 19, 2021. 